\title{
Ferrocarril de La Dorada: configuración, desarticulación y rupturas
}

\author{
Carlos Eduardo Nieto \\ Yenny Andrea Real
}

Introducción

1 Según la descripción que hace Agustín Codazzi, "EI salto llamado Negro o de Honda no es otra cosa que un raudal producido por las rocas i la fuerte inclinación del río, pues en frente de la ciudad, en una distancia de 200 metros, hai una diferencia de nivel de $91 / 2$ metros.

Más abajo de la boca del río Gualí, hasta la Bodega, su desnivel es de 3 metros; arriba del salto, en $150 \mathrm{me}$ tros hai un desnivel de casi

2. De manera que en una distancia de un sesto de miriámetro (1.666 metros) hai un desnivel de $14 \frac{1}{2} 2 \mathrm{me}$ tros, que es lo que produce la fuerza del raudal llamado impropiamente Salto de Honda" (Sánchez, 2014).
Aunque el objetivo de este escrito es realizar una lectura actualizada del conjunto de elementos que conformó el Ferrocarril de La Dorada, ha sido necesario incluir un corto relato histórico introductorio para tener una base de referencia entre el proceso de construcción y el estado actual de conservación del conjunto. Para este efecto, se consultaron fuentes diversas como el Fondo Ferrocarriles Nacionales del Archivo General de la Nación (1907), información documental secundaria y relatos de primera mano. A partir de esta información se realizó la contrastación con el estado actual del sistema, hoy patrimonial.

La unidad de análisis es la línea de $111 \mathrm{~km}$ de longitud, construida entre La Dorada y Ambalema a finales del siglo XIX y principios del XX, la cual evolucionó hasta convertirse en parte fundamental del sistema de interconexión férrea nacional. En este corto tramo se ve reflejada la historia de los ferrocarriles en toda Colombia: las dificultades para su construcción, su crecimiento, esplendor, ocaso y abandono total.

\section{La construcción del Ferrocarril de La Dorada en la historia del país}

La construcción del Ferrocarril de La Dorada nace de la necesidad de dar una solución moderna y mecanizada al principal obstáculo de la navegabilidad del río Magdalena, los llamados "saltos de

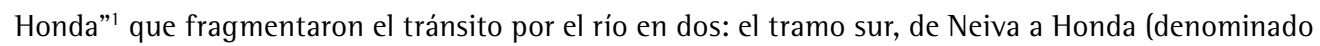
tradicionalmente alto Magdalena) y el tramo norte, de Honda hasta su desembocadura en Bocas de Ceniza (bajo Magdalena).

Con este objetivo, en 1872 el Gobierno Nacional realiza una primera contratación que no se ejecutó por falta de los fondos necesarios para las obras. Solo 10 años más tarde se inician los trabajos y se inaugura la primera milla desde el puerto de Caracolí hacia el sur, buscando el centro de la ciudad de Honda. Al siguiente año se extiende la línea desde el Puerto de Caracolí hacia el norte hasta la quebrada El Peñón y desde el centro de la ciudad de Honda hacia el sur hasta el sitio denominado La Noria, con lo que se completan aproximadamente 5 kilómetros de línea. Con el inicio de la guerra civil colombiana en 1884, quedan detenidos los trabajos del ferrocarril por más de 10 años.

* Cómo citar este artículo: Nieto, C. E. y Real, Y. A. (2017). Ferrocarril de La Dorada: configuración, desarticulación y rupturas. Apuntes, 30(1), 36-53. https://doi.org/10.11144/Javeriana.apc30-1.fdcd 


\title{
Ferrocarril de La Dorada: configuración, desarticulación y rupturas
}

\author{
The Dorada Railway: Configuration, disarticulation and ruptures \\ Ferrovia da Dorada: Configuração, desarticulação e rupturas
}

\section{Carlos Eduardo Nieto}

carlos-nieto@javeriana.edu.co

Arquitecto de la Universidad Nacional de Colombia con estudios de maestría en Filosofía en la Pontificia Universidad Javeriana. Magíster en Restauración de Monumentos Arquitectónicos en la Pontificia Universidad Javeriana. Profesor investigador de la Facultad de Arquitectura y Diseño. Ha sido docente en la maestría en Patrimonio Cultural y Territorio y en el pregrado de Arquitectura tanto en áreas conceptuales como de intervención a diferentes escalas. Investigador en temas relacionados con el manejo integral del patrimonio cultural, y su papel dentro de los estudios territoriales.

Yenny Andrea Real

yreal@javeriana.edu.co

Arquitecta y magíster en Restauración de Monumentos Arquitectónicos de la Pontificia Universidad Javeriana. Actualmente se desempeña como docente en el Área de Historia de la Arquitectura del Departamento de Arquitectura. Publicación del trabajo de grado de la maestría "Paisaje urbano. Estudio patrimonial del eje de la carrera $7^{\text {a }}$ de Bogotá entre el Centro Fundacional y Chapinero", en la colección +1 Arquitectura de Postgrado. Colaboradora y coinvestigadora en investigaciones en temas relacionados con sistemas de información geográfica (SIG) de proyectos de vivienda VIS y de patrimonio industrial colombiano.

Resumen

El presente artículo aborda la dimensión patrimonial de la línea férrea conocida como el Ferrocarril de La Dorada (Colombia, departamentos de Caldas y Tolima) a partir de dos aspectos básicos: el primero tiene que ver con el reconocimiento de la construcción de este ferrocarril como un sistema territorial complejo; y el segundo, el contraste con el estado actual de los elementos de función especializada dentro de la línea de $111 \mathrm{~km}$ de longitud, principalmente de arquitectura y de ingeniería. Para esto, el análisis del Ferrocarril de La Dorada como una unidad patrimonial parte en un primer momento de la recopilación y análisis del proceso histórico de conformación de esta línea, para luego definir los elementos constitutivos del sistema. En un tercer momento, se hace un recorrido y registro del estado actual de los elementos más importantes definidos, y se culmina con unas notas sobre las necesidades de articulación para su conservación.

Palabras clave: patrimonio ferroviario; patrimonio industrial; territorio; paisaje cultural; inventarios

\section{Abstract}

This article deals with the patrimonial dimension of the railroad known as the La Dorada railroad, based on two basic aspects: the first one deals with the recognition of the construction of the railway as a complex territorial system; and the second, the contrast deals with the current state of the specialized-function elements along the $111 \mathrm{~km}$ long line, mainly in terms of architecture and engineering. For this purpose, the analysis of the La Dorada railroad as a heritage unit starts at the compilation and analysis of the historical conformation process of this line, and proceeds to the definition of the constituent elements of the system. Then, we carry out a tour and record of the current state of the most important elements defined, to conclude with some notes on articulation needs for its preservation.

Keywords: Railroad heritage; industrial heritage; territory; railroad landscape; inventories

Artículo de investigación

Recepción: 28 de agosto de 2016

Aceptación: 30 de noviembre de 2016

Disponible en línea: 10 de diciembre de 2017
Resumo

O presente artigo aborda a dimensão patrimonial do transporte pela ferrovia conhecida como Ferrocarril de La Dorada, a partir de dois aspectos básicos: 0 primeiro tem a ver com o reconhecimento da construção do transporte ferroviário como sistema territorial complexo; e o segundo, com o contraste com o estado atual dos elementos de função especializada dentro da ferrovia de $111 \mathrm{~km}$ de largura, principalmente de arquitetura e engenharia. Para isso, a análise da Ferrovia de La Dorada como unidade patrimonial parte em um primeiro momento da coleção e análise do processo histórico de conformação desta via, para definir os elementos constitutivos do sistema. Em um terceiro momento, faz-se um percurso e registro do estado atual dos elementos mais importantes definidos, para acabar com anotações sobre as necessidades de articulação para a sua conservação.

PALAVRAS-CHAVE: Patrimônio ferroviário; patrimônio industrial; território; paisagem ferroviária; inventários doi:10.11144/Javeriana.apc30-1.fdcd 
Solo hasta 1894 se retoman los trabajos en la línea del Ferrocarril de La Dorada, dando al servicio un nuevo tramo que va desde el sitio denominado Perico hasta el puerto de Arrancaplumas (al sur de Honda). Un año más tarde (1895), se extiende la línea hacia el norte hasta el punto Yeguas, en un meandro apto para el desembarco, completando $22 \mathrm{~km}$ de línea aproximadamente.

La línea del ferrocarril llega finalmente al puerto de La María (posteriormente llamado La Dorada) en el año de 1897, con el que se sobrepasa el objetivo inicial de sortear los "saltos de Honda" pues con una longitud total de 32 kilómetros entre La Dorada y Arrancaplumas, se evita además un tramo de navegabilidad difícil e inestable entre La Dorada y Honda. Este corto tramo tardó 25 años en su construcción, con un promedio de ejecución de 1,28 km/año y contó con las siguientes estaciones:

\begin{tabular}{|l|l|}
\hline $0 \mathrm{~km}$ & La Dorada \\
\hline $7 \mathrm{~km}$ & Purnio \\
\hline $16 \mathrm{~km}$ & Guarinocito \\
\hline $23 \mathrm{~km}$ & Perico (paradero) \\
\hline $32 \mathrm{~km}$ & Honda \\
\hline
\end{tabular}

Ya desde 1891 se había determinado la necesidad de la ampliación del Ferrocarril de La Dorada hacia el sur, de Arrancaplumas hasta un puerto en inmediaciones de Ambalema o, eventualmente, hasta Girardot, y así mejorar este corredor surnorte. Las obras de este nuevo tramo se iniciaron de manera efímera en 1899, pues las paralizó la Guerra de los Mil Días y solo se retomaron en 1905. Esta prolongación se dio al servicio en 1907 completando un total de 111 kilómetros de línea del Ferrocarril de La Dorada hasta Ambalema. Esta segunda fase tardó dos años en su construcción, con un promedio de $39,5 \mathrm{~km} /$ año.

Con el sistema del ferrocarril en crecimiento en todo el país, el Ferrocarril de La Dorada tuvo desde 1907 un funcionamiento constante y sostenido, lo que permitió su desarrollo técnico y su fuerte incidencia territorial. Este auge se reafirmó con la entrada en funcionamiento del cable aéreo Mariquita-Manizales ${ }^{2}$, en 1922, que inyectó un flujo adicional de carga a la línea, principalmente de café de exportación.

Con las sucesivas ampliaciones de la línea, primero hacia el sur y su interconexión con los ferrocarriles de lbagué, de Girardot y de Huila; y luego hacia el norte, que en la década de los años sesenta se interconectaría con el del Magdalena para conformar la "Ruta del Sol”, el tramo original del Ferrocarril de La Dorada aumentó de manera sostenida su impacto territorial al
2 "Los trabajos comenzaron el 2 de septiembre del 1913, entre Manizales y Mariquita, en una longitud de 73 km $361 \mathrm{~m}$, alcanzando su mayor altitud 3.675 $\mathrm{m}$, en el km 51.815. La obra quedó totalmente terminada en 1921" (Bateman, 2005).

Figura 1:

Cronología de la

construcción del

Ferrocarril de La Dorada

Fuente:

Imagen elaborada por los autores. Itinerario tomado de Ortega, 1911
Aกั้

1872 Se establece el

Primer contrato

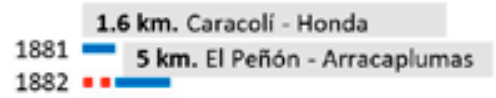

1882

Guerra Civil Colombiana (1884 -1885)

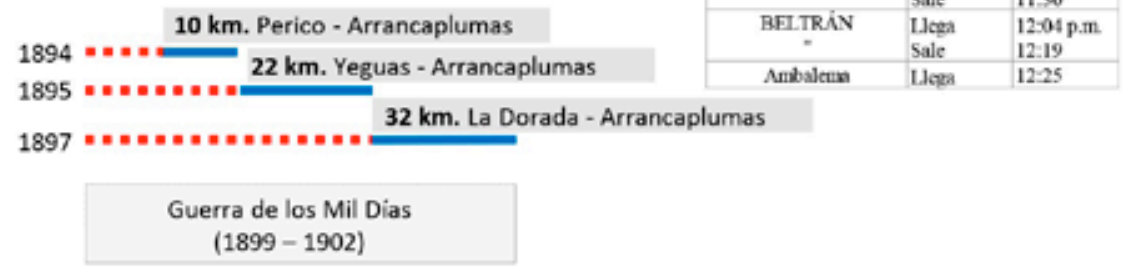

111 km. La Dorada - Ambalema

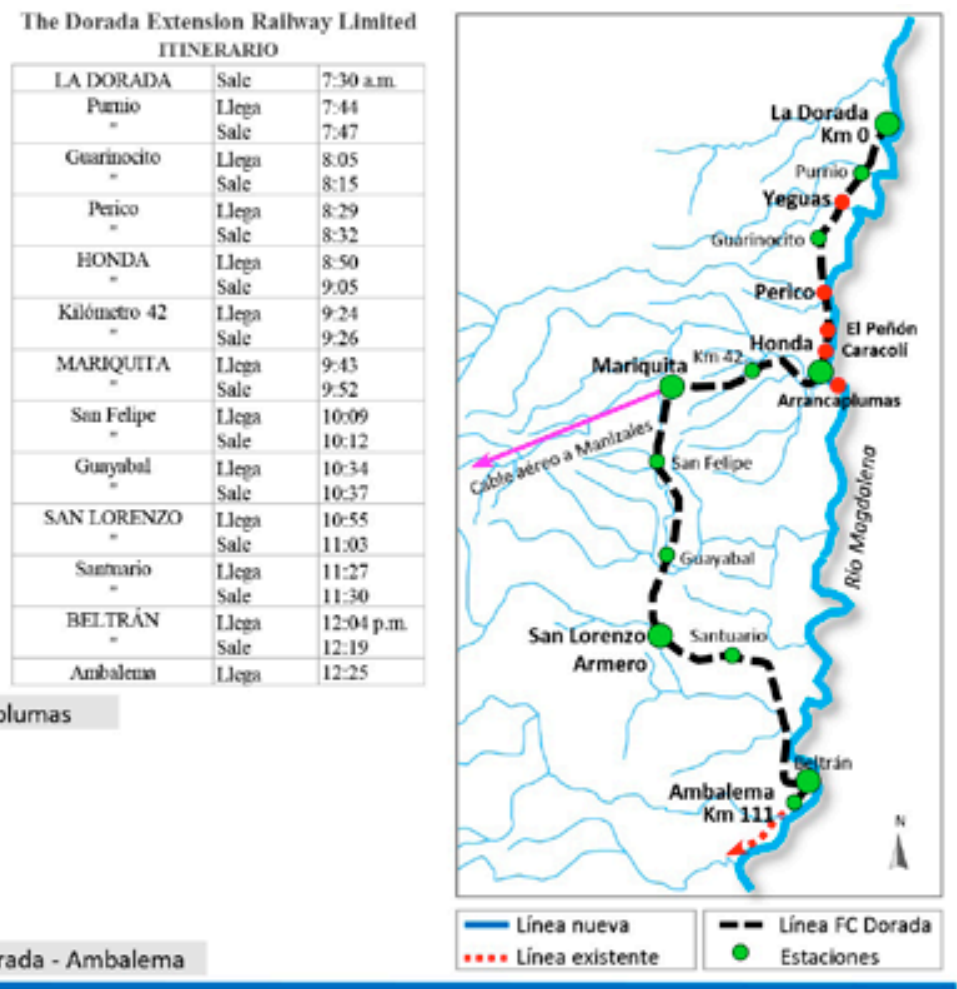

$\begin{array}{cccccccccccccccccccccccc}1 & \\ \text { Kilómetro } & 5 & 10 & 15 & 20 & 25 & 30 & 35 & 40 & 45 & 50 & 55 & 60 & 65 & 70 & 75 & 80 & 85 & 90 & 95 & 100 & 105 & 110\end{array}$


articular el movimiento de carga y pasajeros de las zonas más productivas y más densamente pobladas del país.

Tras una historia de aproximadamente 100 años de servicio y desarrollo, y a pesar de ser un tramo central en el sistema férreo nacional, el paso de locomotoras por esta línea fue suspendido hacia 1980, cuando quedó en desuso todo el sistema. Sus elementos constitutivos se debaten hoy entre el olvido, el desmantelamiento por vandalismo, la ocupación ilegal y la pérdida de su materialidad. Hasta la fecha no se ha logrado la puesta en marcha de mecanismos eficaces para su reestructuración dentro de las nuevas demandas de la economía regional y nacional.

La noción de sistema aplicada al Ferrocarril de La Dorada

El funcionamiento del ferrocarril, aun desde sus versiones más elementales a finales del siglo XIX, exigió una organización sistémica de elementos con funciones específicas, todos al servicio de un objetivo primordial: el transporte mecanizado y eficiente de mercancías y personas, a partir del uso de locomotoras sobre un camino de rieles. Esta premisa reconoce la presencia y funcionamiento armónico de diversos elementos en el espacio geográfico alrededor de dos funciones básicas: circulación (líneas) y transferencia (nodos). Adicionalmente, aparece una tercera función complementaria que dio soporte administrativo y técnico a las dos primeras.

Para la circulación del equipo rodante por las líneas férreas se tuvieron que solucionar problemas técnicos asociados a la quebrada topografía y la calidad de los suelos. Estas condiciones propiciaron la aparición en el paisaje de los terraplenes que daban sustento adecuado a los rieles y corredores, y otros elementos más específicos de gran valor histórico y técnico como túneles, puentes, viaductos, pasos a nivel, alcantarillas, pasos de escorrentías, etc. Todos estos elementos tenían la función específica de darle continuidad a las líneas de transporte, previa e intencionalmente trazadas y construidas.

Para las funciones de transferencia, la variedad de elementos desarrollados fue mucho mayor. Esta variedad da cuenta de manera más amplia de las condiciones del territorio: estaciones, bodegas, triángulos de escape, tornamesas de volteo, puertos, complejos industriales de transformación dependientes del sistema del ferrocarril, etc. En esta función, la multimodalidad fue la característica más importante del desarrollo del ferrocarril en Colombia, en tanto los nodos de transferencia fueron en gran medida sitios de cambio de modo de transporte: entre camino de herradura, río navegable, cable aéreo o ferrocarril.

Las actividades complementarias, como tercera función, en primera instancia dieron soporte administrativo a través de la construcción de oficinas y viviendas para el personal de apoyo. En segundo término y de manera similar, para el soporte técnico se crearon talleres para el equipo rodante, almacenes, fábricas de repuestos, talleres de fundición y campamentos para las cuadrillas de mantenimiento de las líneas, entre otros.

La eficiencia del ferrocarril dependía, en buena parte, de la forma oportuna en que estos elementos interactuaban y cumplían sus funciones especializadas, y de la forma como el sistema se articulaba a las condiciones de los territorios que atravesaba.

En el informe presentado al Ministerio de Obras Públicas en 1911 (Ortega, 1911) se realizaba un inventario riguroso de la línea del Ferrocarril de La Dorada en el que se clasificaban los elementos del sistema en: obras de arte (puentes, pontones, alcantarillas y desagües), estaciones, talleres, bodegas, oficinas y obras accesorias (tanques de agua y obras de consolidación de orillas).

A partir de la categorización propuesta por este informe, la investigación estructuró la clasificación base que guio el trabajo de campo ${ }^{3}$ con el fin de verificar la permanencia de estos elementos en la actualidad. Adicionalmente, se valoró de manera preliminar su estado de conservación y se reconocieron las articulaciones territoriales de la línea. Para esta aproximación a los elementos se definieron las siguientes categorias:

- Puentes: son estructuras de longitud mayor a 3 metros que soportan la función de circulación. Aquellas de menor longitud, correspondientes a escorrentías, alcantarillas y desagües no se tuvieron en cuenta. Para la calificación del estado de conservación se tuvo en cuenta la estabilidad o integridad de sus tres elementos principales: 1. las bases o estribos, 2. la estructura principal del puente $y, 3$. las 
traviesas, rieles y barandas. Esta calificación se realizó a vista, sin evaluación de resistencia o degradación de materiales. Tras 35 años de desuso, intemperie y nulo mantenimiento es muy probable que la capacidad portante de estas estructuras esté por debajo del mínimo necesario para su reutilización.

- Estaciones: corresponden a la función de transferencia. Son edificaciones destinadas al movimiento de pasajeros y a carga y descarga de mercancías menores, con mínimas anexidades: bodega y/o servicios. Para la calificación del estado de conservación se tuvieron en cuenta tres indicadores básicos: 1 . Estabilidad e integridad de los muros exteriores, 2. Estado general de la cubierta del edificio principal y, 3. Estado de los acabados, carpintería, pintura exterior.

- Bodegas: corresponden a la función de transferencia. Son edificaciones aisladas que no estuvieron asociadas a una estación de pasajeros por estar dedicadas exclusivamente al almacenaje temporal de mercancías.

- Campamentos: corresponden a la función complementaria. Son edificaciones aisladas construidas exclusivamente para albergar las cuadrillas de mantenimiento de la línea. Estas edificaciones no estuvieron relacionadas con las estaciones de pasajeros.

- Conjuntos: se denomina conjunto a aquellos nodos que, por su importancia dentro de la línea y por su capacidad de relación con el territorio circundante, complementan la función primaria de la estación de pasajeros con varios tipos de funciones (de transferencia y de uso complementario). Esto implicó el desarrollo de edificaciones anexas a la estación de pasajeros como: oficinas, bodegas, talleres, campamentos, corrales, un patio de maniobras de mayor proporción.

En la calificación del estado de conservación de las bodegas, campamentos y conjuntos, se tuvieron en cuenta las mismas consideraciones que para las estaciones.

Las oficinas y obras accesorias no se tuvieron en cuenta por su baja incidencia en las dinámicas actuales, pero se incluyó un cruce o triángulo de escape que funcionó originalmente como la bifurcación entre la línea hacia Ambalema y la línea original hacia Arrancaplumas, en el casco urbano de la ciudad de Honda.
El consolidado de los elementos registrados es el siguiente:

\begin{tabular}{|l|c|}
\hline Puentes & 28 \\
\hline Estaciones & 11 \\
\hline Bodegas & 4 \\
\hline Campamentos & 2 \\
\hline Conjuntos & 4 \\
\hline Cruce & 1 \\
\hline Total & 50 \\
\hline
\end{tabular}

Caracterización de la línea del Ferrocarril de La Dorada

La evolución del Ferrocarril de La Dorada conformó y transformó diferentes unidades territoriales, generando funciones sistémicas en cada una de las zonas por las cuales transitó. Tras la revisión de las condiciones geográficas e históricas y el trabajo de campo, se subdividió la línea de $111 \mathrm{~km}$ en cinco tramos con características y soluciones específicas. Cada uno de estos tramos genera una unidad homogénea que, si bien no genera diferencias en aspectos tipológicos o constructivos, sí produce formas diferentes de relación con el territorio.

- Tramo 1: sorteando meandros. Desde el conjunto La Dorada a estación Perico. Km 0 a $\mathrm{km}$ 23. En el tramo se registraron 1 conjunto, 2 estaciones, 1 bodega, 1 campamento y 6 puentes, para un total de 11 elementos.

- Tramo 2: siguiendo el río. De la estación Perico a bodegas Caracolí. Km 23 a km 29, se registraron 1 estación y 3 puentes, para un total de 4 elementos.

- Tramo 3: origen y ramal. Comprende desde las bodegas Caracolí al campamento Calunga, en el casco urbano de Honda. Km 29 a $\mathrm{km} \mathrm{37,} \mathrm{donde} \mathrm{se} \mathrm{encontraron} 1$ conjunto, 3 bodegas, 6 puentes y 1 cruce, para un total de 11 elementos.

- Tramo 4: hacia la cordillera. Del campamento Calunga a Armero (estación San Lorenzo). $\mathrm{Km} 37 \mathrm{a} \mathrm{km} \mathrm{79,} \mathrm{se} \mathrm{encontraron} 1$ conjunto, 4 estaciones, 1 campamento y 10 puentes, para un total de 16 elementos.

- Tramo 5: de vuelta al río. Desde Armero (Estación San Lorenzo) a Ambalema. Km 79 a $\mathrm{km}$ 111. En este tramo se encontró 1 conjunto, 4 estaciones y 3 puentes para un total de 8 elementos. 
Figura 2:

Tramos Ferrocarril

de La Dorada

Fuente:

Imagen elaboradas

por los autores

Figura 3: Tramo 1 - Ferrocarril de La Dorada

Fuente:

Imagen y fotografías elaboradas por los autores

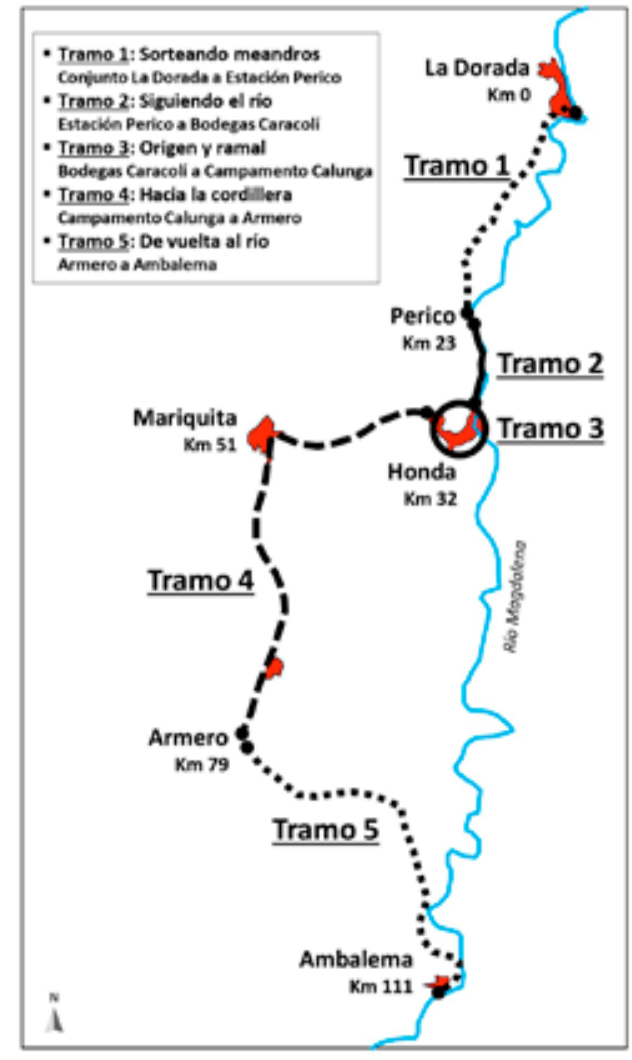

Tramo 1: sorteando meandros, conjunto La Dorada a estación Perico

Este primer tramo comprende desde el conjunto de La Dorada, kilómetro 0, hasta llegar a la estación de Perico en el kilómetro 23, su línea férrea recorre una topografía plana, con paisaje abierto hacia el río Magdalena, y una parte montañosa hacia el occidente. En esta zona el río forma una serie de meandros que restringen la navegabilidad y que han cambiado su curso de manera notoria a lo largo de los años. Para sortearlos, la vía férrea se aleja del río, teniendo contacto directo con este solo en dos puntos: el primero en La Dorada y el segundo en el sitio denominado la Vuelta de Yeguas, donde funcionó uno de los puertos provisionales a finales del siglo XIX.

A la desembocadura del río Guarinó la línea férrea llega muy cercana a la ribera del Magdalena, y se ve afectada por la constante socavación de su orilla norte, lo que generó múltiples obras de ampliación del puente hacia

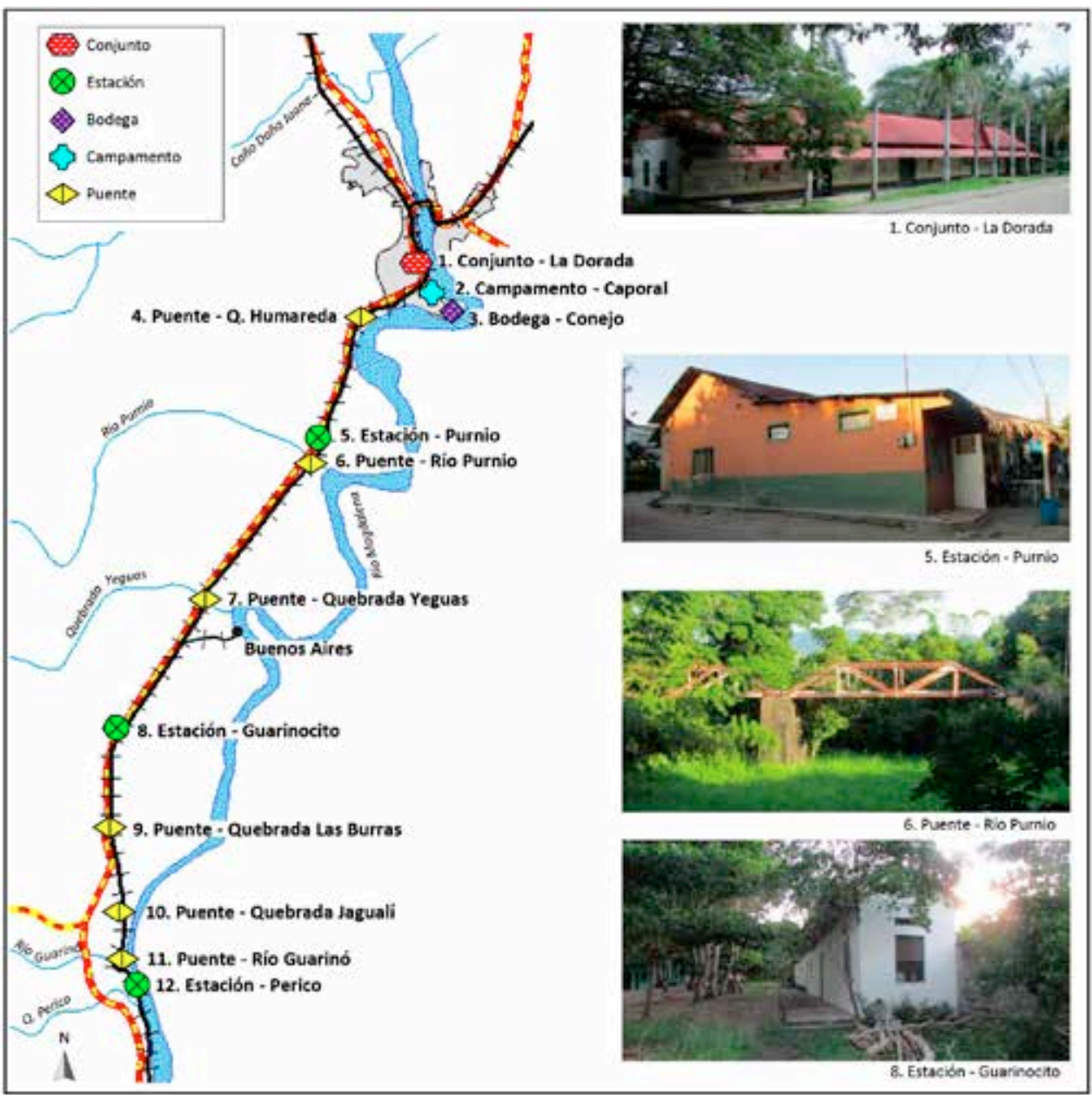




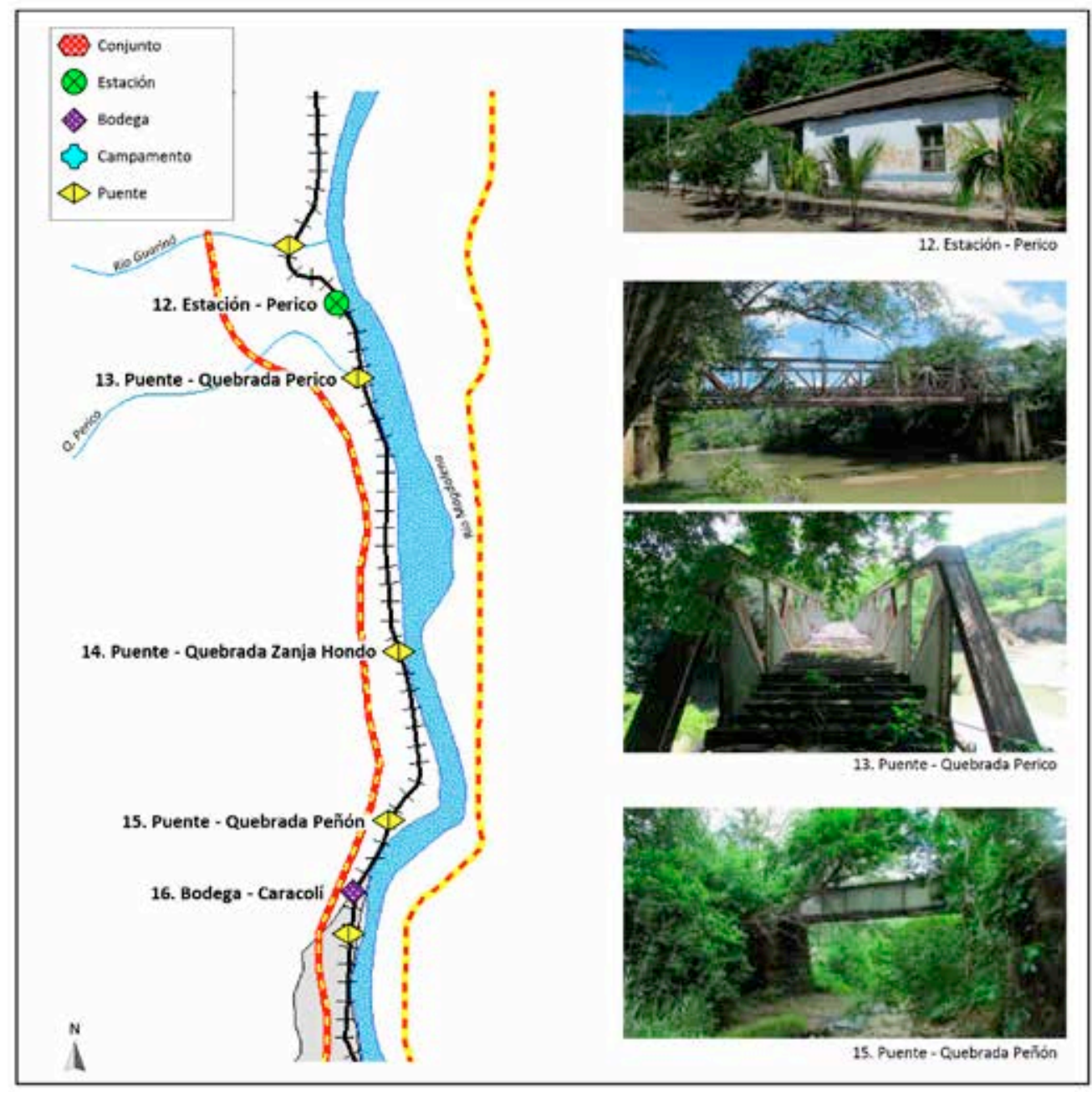

ese costado. En este tramo el Magdalena tiene 6 afluentes: quebrada Humareda, río Purnio, quebrada Yeguas, quebrada Las Burras, quebrada Jagualí y río Guarinó. En cada uno de estos puntos se construyeron puentes que van de los 10 a los 170 metros de luz, como es el caso del puente sobre el río Guarinó que constituye la estructura de este tipo más larga de todo el Ferrocarril de La Dorada.

Respecto a las articulaciones territoriales, cabe mencionar que en el casco urbano de La Dorada se encuentra la estación México, punto de partida de la línea que interconecta con el norte del país a través del Ferrocarril del Magdalena. Las estaciones La Dorada y México están articuladas por un tramo de línea férrea. Además, se encuentra el puente Ferry Atlántico que sirve de conexión con la población de Puerto Salgar donde se inicia la ruta del Ferrocarril de Cundinamarca que conduce a la Sabana de Bogotá.

Por otra parte, el tramo tiene un funcionamiento de corredor (tren y vía vehicular), con puntos de contacto hacia el río, pero sin conexiones hacia el occidente. Al revisar la articulación territorial con las carreteras actuales, se observa que se desarrollaron siguiendo el trazado de la vía férrea, exceptuando la zona urbana de La Dorada y el sector de la desembocadura del río Guarinó, donde el trazado del ferrocarril y la carretera se alejan considerablemente.

La estación de pasajeros de La Dorada, ubicada en el casco urbano, marcó el inicio del tramo y era la parada más importante de un subsistema (dentro del perímetro urbano), fue complementada con la actividad del campamento El Caporal, una edificación menor que prestó funciones de control de tráfico, y estancia para cuadrillas de mantenimiento; y con la del puerto y bodegas de Conejo, ubicadas a 1 kilómetro de la estación en línea recta hacia el suroriente, sitio en el cual "el río Magdalena ha hecho cambios importantes en el meandro frente al barrio Bucamba, circunstancia que llevó a considerar a principios del siglo XX el
Figura 4:

Tramo 2 - Ferrocarril de La Dorada Fuente: Imagen y fotografías elaboradas por los autores 
4 En este sitio, el cauce del río cambió naturalmente, al variar la antigua "Vuelta de la Madre de Dios, gran meandro que formaba el Magdalena a cinco leguas granadinas $(25 \mathrm{~km})$ a norte de Honda" (Sánchez, 2014) conformó la actual charca de Guarinocito.

Figura 5:

Casco urbano de Honda en 1941, con la ubicación de los elementos del Ferrocarril de La Dorada

Fuente:

Elaboración de los autores sobre aerofotografía del Instituto Geográfico Agustín Codazzi, Bogotá lugar, como 'punto cero' de la navegación del río" (Duque, 2013).

La estación de pasajeros se complementa con un total de cinco bodegas dispuestas en doble línea a lado y lado de la línea férrea. Hoy en día la estación de pasajeros está ocupada por algunas dependencias de la alcaldía, las bodegas del conjunto están en desuso, el campamento El Caporal fue adaptado para vivienda y en la única bodega que sobrevive del antiguo conjunto de Conejo, funciona un punto de acopio de reciclaje.

La segunda parada es en el centro poblado de Purnio, la estación se ubica al norte del río del mismo nombre y constituye el punto central del poblado que se ha conformado alrededor de la estación y que ha crecido linealmente sobre el corredor férreo. La última parada de este primer tramo se ubica en la estación de Guarinocito, a una cuadra de distancia de la plaza principal, en el borde oriental del centro poblado adyacente a la charca del mismo nombre ${ }^{4}$. Estas dos últimas estaciones, la de Purnio y Guarinocito, fueron adaptadas para el uso de vivienda y comercio informal.
Por efecto del desuso y la falta de mantenimiento general de la línea, el tramo presenta pérdida total del puente Humareda, y pérdida parcial en los puentes Jaguali y Guarinó. Estas pérdidas implican una interrupción total de la continuidad de la línea férrea lo que ha estimulado la sustracción de rieles y demás elementos complementarios de la línea. En general los inmuebles de las estaciones de pasajeros están en buen estado de conservación a excepción de sus acabados, pintura y carpintería. A pesar de las condiciones señaladas, el tramo guarda un alto nivel de integridad si se toman consideraciones como la integración del paisaje, la conservación de los terraplenes y el respeto y bajo nivel de invasión del corredor férreo.

\section{Tramo 2: siguiendo el río, estación Perico a bodegas Caracolí}

El segundo tramo está definido desde la estación Perico en el kilómetro 23, hasta las bodegas de Caracolí, kilómetro 29, punto de entrada a la ciudad de Honda. La línea férrea recorre topografía plana, con paisaje montañoso hacia el

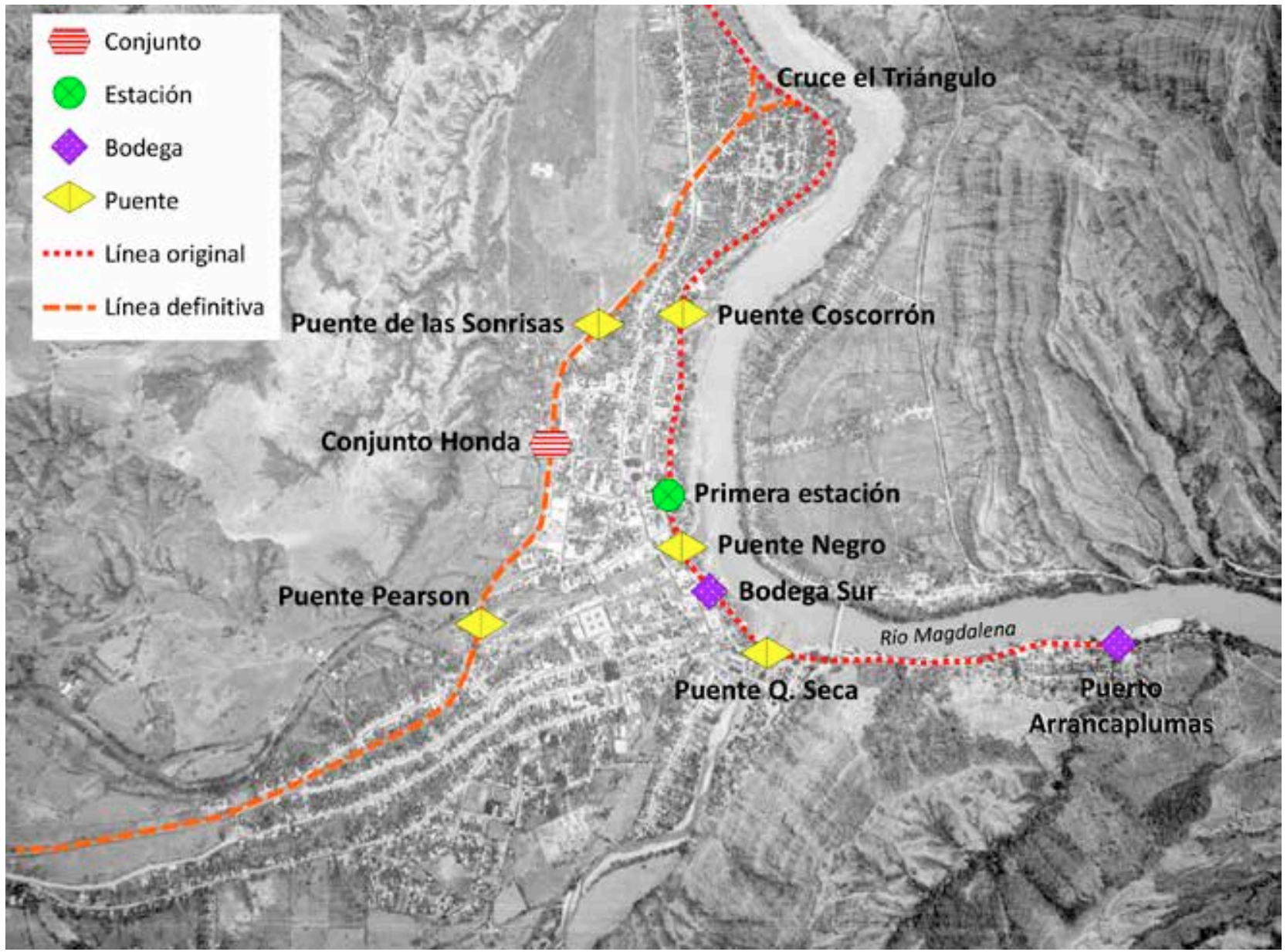


occidente y abierto hacia el río Magdalena. En este tramo el río corre linealmente, sin variaciones importantes en su curso, con afluentes menores, entre los cuales sobresale la quebrada Perico. La línea férrea y la actual carretera siguen muy de cerca el curso del río, excepto la zona de la estación Perico, donde las dos vías se alejan aproximadamente 1 kilómetro. En el punto de la estación Perico confluye la vía que conecta con la zona centro oriente del departamento de Caldas, municipios de Victoria, Marquetalia y otras poblaciones menores, y se constituye en el punto de articulación territorial más importante de este tramo.

La única estación que se encuentra en este tramo es la de Perico, en un contexto rural no urbanizado, a una distancia aproximada de 50 metros sobre la margen izquierda del río Magdalena. La línea férrea cruza tres quebradas, con tres puentes: Perico, Zanja Hondo y Peñón, con luces que van de 5 a 40 metros. Según Poveda (2010), hasta el puente de la quebrada el Peñón,
700 metros arriba de las bodegas de Caracolí, podían llegar los vapores del bajo Magdalena sin enfrentarse con los violentos raudales del salto de Honda.

En general, los elementos de este tramo se encuentran en buen estado. La estación Perico no se usa, pero tiene en buen estado de conservación, a excepción de los acabados, pintura y carpintería. La estructura de los tres puentes aún se conserva, aunque las traviesas y los rieles han desaparecido parcialmente.

\section{Tramo 3: origen y ramal, bodegas Caracolí a campamento Calunga}

Este tercer tramo está definido desde las bodegas de Caracolí, en el kilómetro 29, hasta llegar al campamento Calunga, kilómetro 37 aproximadamente. En este tramo la línea férrea recorre la ciudad de Honda en dos direcciones: la primera, el trazado original que pasaba por el centro de la ciudad y llevaba al puerto de

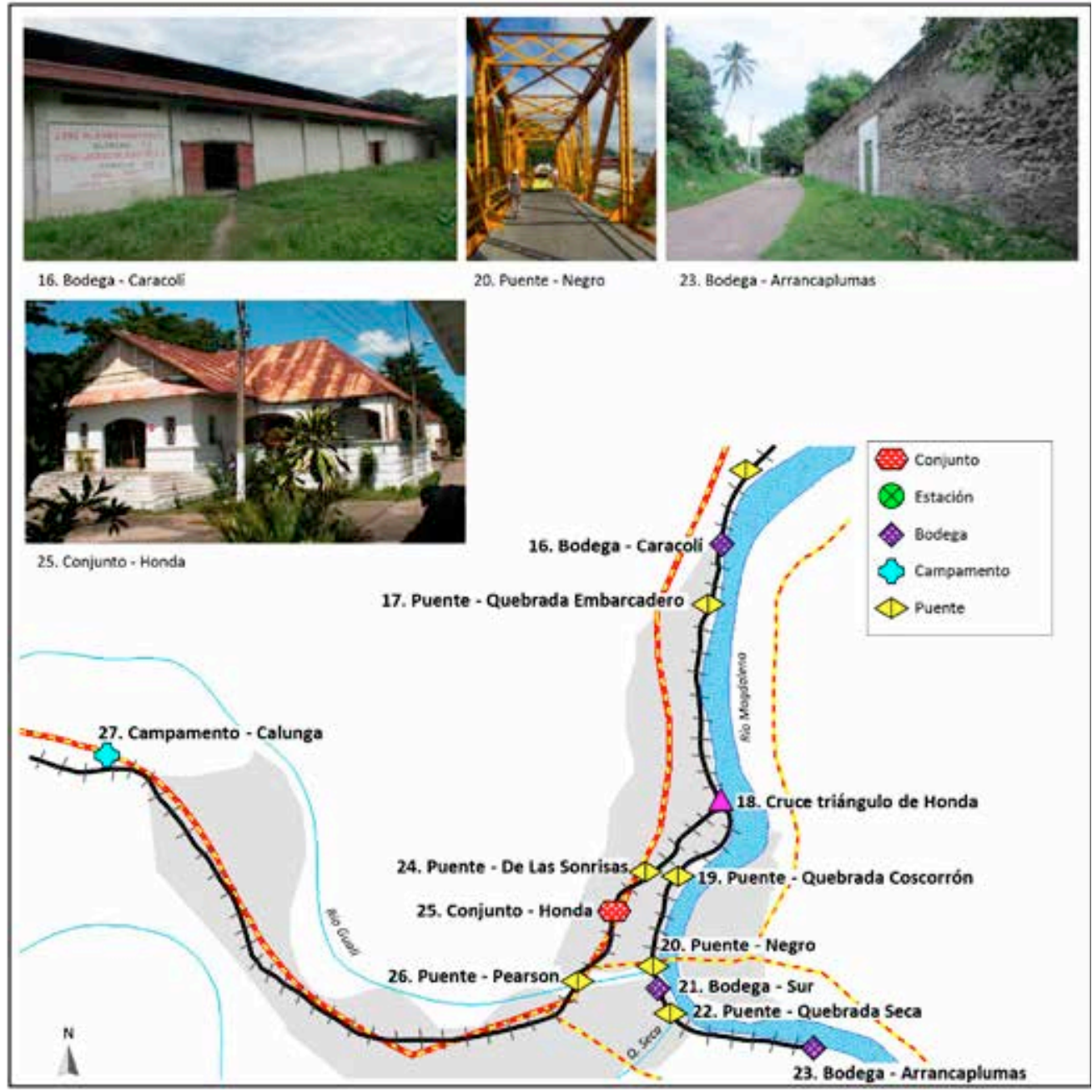

Figura 6:

Tramo 3 - Ferrocarril de La Dorada

Fuente:

Imagen y fotografías elaboradas por los autores 
Arrancaplumas, y la segunda, que sigue la prolongación hacia Ambalema y desvía pasando por la actual estación de San Juan de Dios, atraviesa el Gualí por el desaparecido puente Pearson y toma el curso del río Gualí hacia Mariquita.

Los saltos de Honda caracterizan este tramo en su calidad de elemento natural ordenador de la lógica territorial y de la configuración urbana de Honda. El ferrocarril acopló su estructura funcional y técnica a esta determinante superior del territorio, pero los cambios en el trazado generaron dos formas de funcionamiento que conviven en este tramo: la primera es la de punto terminal de línea e interconexión inmediata con el tráfico a Bogotá (llegada al puerto de Arrancaplumas), lo que generó una serie de edificaciones o sitios con relaciones sistémicas entre sí, fundamentales en la configuración urbana de Honda. La segunda forma, que se traslapa y complementa con la primera, es la de un tramo de paso que genera un nodo funcional muy importante, pero que se aleja de la dinámica y paisaje del Magdalena en busca de una relación territorial con las zonas productivas de la Cordillera Central (ampliación hacia Mariquita).

Honda, denominada en la época colonial como la "garganta del reino", y luego cruce de caminos, estrella de comunicación y otros apelativos que señalan esa vocación, fue el nodo más relevante del Ferrocarril de La Dorada desde finales del siglo XIX. El ferrocarril articuló el funcionamiento general de la ciudad, lo que reforzó su vocación comercial y de cruce de caminos, y por su parte, la ciudad fue el punto base de la actividad ferroviaria. En Honda "se formaron dos terminales para los vapores: el de Caracolí, al norte de la ciudad, para el bajo Magdalena; y el de Arrancaplumas, al sur de la ciudad para los buques del alto Magdalena" (Poveda, 2010), para poder sortear el paso de pasajeros y productos de importación y exportación por los saltos de Honda. En su paso por la ciudad, el ferrocarril generó bodegas especializadas en productos de comercio nacional e internacional, estación de pasajeros, oficinas de la Compañía

5 Según el Centro de Historia de Honda, el puente Navarro inició su construcción en 1894, fue terminado el 24 de diciembre de 1898 e inaugurado el 16 de enero de 1899. Bernardo Navarro adquirió la concesión del puente con cobro de peaje por un lapso de 99 años. la aparición de una nueva estación en el sector periférico, sobre el trazado de la prolongación hacia Mariquita y Ambalema. Con este desplazamiento, la línea del ferrocarril abandonó el curso del río Magdalena y tomó el del río Gualí que marcó el borde occidental de la ciudad.

La actual estación de pasajeros de Honda, ubicada en el sector de San Juan de Dios, está compuesta por un conjunto de aproximadamente seis edificaciones dedicadas a funciones de transferencia y complementarias, un espacio abierto cercado usado como embarcadero de ganado y un amplio patio de operaciones. Está actualmente ocupada por una familia que adecuó parte de su planta como vivienda. Se encuentra en buen estado de integridad, con problemas visibles de falta de mantenimiento en toda su construcción.

La antigua estación de pasajeros, primera parada urbana en la línea que conduce a Arrancaplumas, corresponde al actual Centro Comercial Santa Mónica, en donde además funcionó la oficina principal de la Compañía, funciones de las cuales no queda ninguna huella legible en la actualidad.

En cuanto a las bodegas ubicadas en este tramo, se encuentran inicialmente las de Caracolí, antiguo puerto localizado aproximadamente a 3 kilómetros al norte del centro histórico de Honda, en la periferia urbana. Constituye un conjunto de bodegas con muelle sobre el río, que señala una actividad importante de carga mas no de pasajeros, pues no existió un edificio dedicado a esta función. Luego se encuentra la llamada bodega Sur, que corresponde a las antiguas bodegas ubicadas al sur del río Gualí y muy cerca de la ribera del Magdalena, destinada originalmente para el almacenaje de mercancías de importación y exportación. Cabe anotar que se encontraron menciones sobre la existencia de otra bodega al norte del río Gualí, contigua a la estación de pasajeros, de la cual no se logró definir su ubicación exacta. Las bodegas de Arrancaplumas constituyen el vestigio del antiguo puerto, y están ubicadas aproximadamente a 1,5 kilómetros al suroriente del centro histórico de Honda, en la periferia urbana. Respecto al uso actual de las bodegas registradas en la ciudad de Honda, las de Caracolí y Arrancaplumas están desocupadas y en la bodega Sur funciona una trilladora.

En el lugar geográfico donde se ubicó la

ciudad de Honda, confluye la desembocadura zado paulatinamente del centro de la ciudad, con y puentes ferroviarios que impactaron todo e sector central de la ciudad. Esta dinámica se vería complementada con la construcción y puesta en servicio del puente Navarro ${ }^{5}$, primer puente metálico sobre el Magdalena.

El movimiento del ferrocarril sería despla- 


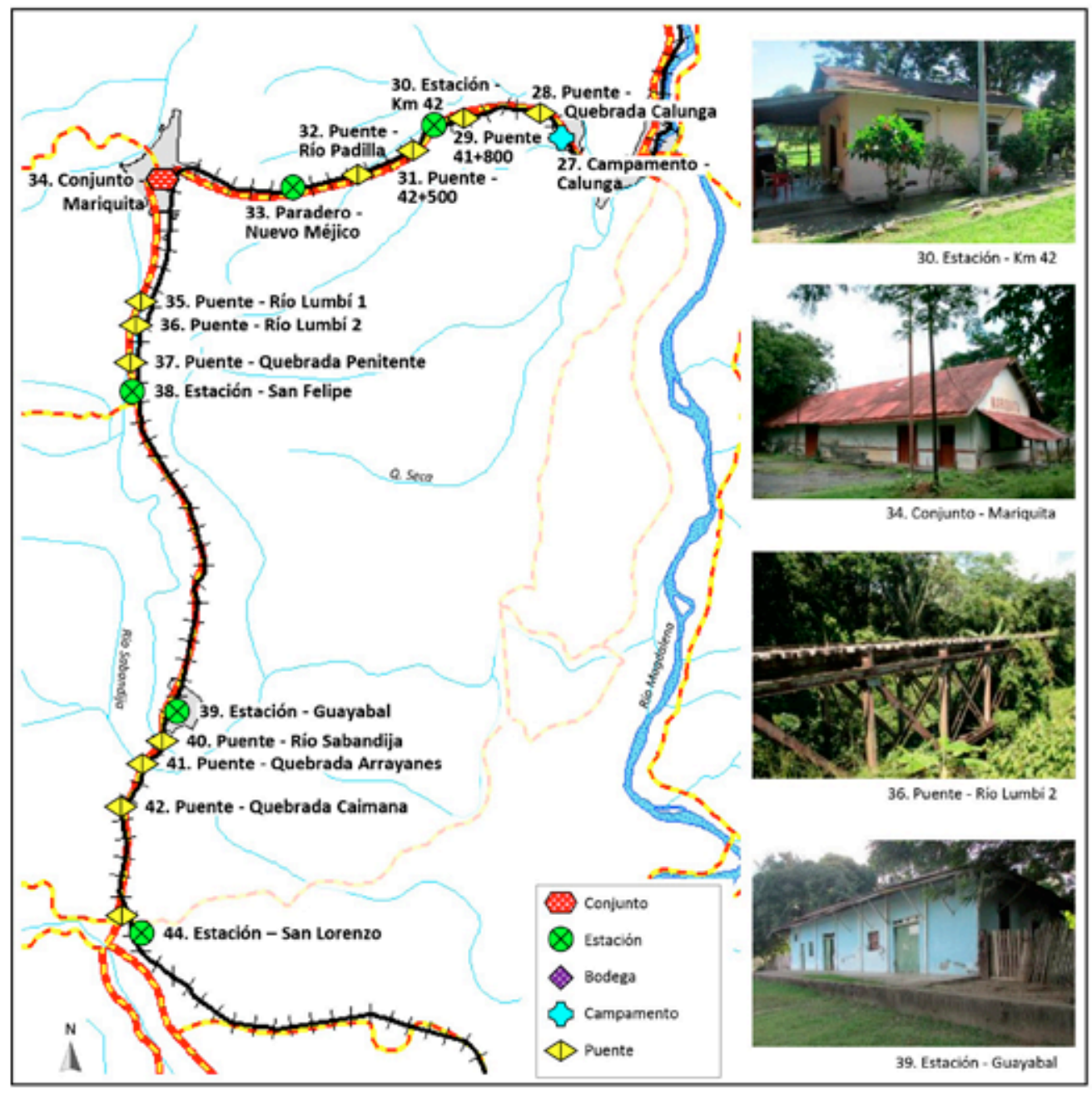

del río Gualí y la quebrada Seca en el río Magdalena, condición que fragmenta el territorio de la ciudad y genera la necesidad de puentes para poder mantener la continuidad de las líneas férreas. Por este motivo se construyeron un total de seis puentes de origen férreo de los cuales el primero está sobre la quebrada el Embarcadero, ubicado a 300 metros al sur de las bodegas de Caracoli; tres puentes dentro del casco urbano que corresponden a la línea original hacia Arrancaplumas: puente quebrada Coscorrón, puente Negro y puente quebrada Seca; y los otros dos puentes, también en casco urbano, que corresponden al trazado de la prolongación: puente de las Sonrisas y puente Pearson. Este último, la estructura más imponente de toda la línea, tenía 118 metros de largo y 60 metros de altura y se derrumbó por la caída de su base sur en una creciente del río, el 13 de enero de 2006.

En la medida que el ramal a Arrancaplumas dejó de funcionar tempranamente, sus elementos constitutivos se perdieron, bien, por desaparición física, o bien, por absorción de las dinámicas de la zona urbana de la ciudad. En esta línea desaparecieron dos de tres puentes, y el tercero (Coscorrón) hace parte de la estructura de la vía vehicular. De la misma manera, las edificaciones (bodegas y estación antigua) han perdido su legibilidad dentro del conjunto urbano. Sobre la línea de la prolongación se encuentra el puente de las Sonrisas en buen estado y adaptado a la estructura vial de la ciudad, pero en contraste, el puente Pearson constituye el vacío más importante.

\section{Tramo 4: hacia la cordillera, campamento Calunga a Armero}

El cuarto tramo está definido desde el campamento Calunga en el kilómetro 37, hasta la estación San Felipe, en la desaparecida población de Armero en el kilómetro 79. La línea férrea se aleja abiertamente del río Magdalena y su dinámica en busca de la conexión con Mariquita,
Figura 7:

Tramo 4 - Ferrocarril de La Dorada

Fuente: Imagen y fotografías elaboradas por los autores. 
y para esto recorre un territorio relativamente plano, con un paisaje abierto y montañoso hacia los dos lados de la línea. A partir de Mariquita la línea toma hacia el sur y vuelve a ser paralela al río Magdalena, pero a una distancia media de 20 kilómetros hasta llegar a la población de Armero. Este movimiento hacia la cordillera obedece a la necesidad de conexión de esta zona occidental del país, históricamente aislada por las dificultades que ofrece su compleja topografía. El tramo tiene un funcionamiento de corredor estructural de la red, sin conexiones hacia el río, pero con gran conectividad con carreteras que unen con el occidente del país.

En este tramo se entrecruzan constantemente la vía férrea y el desarrollo de las carreteras actuales: la carretera principal Honda-Armero, que se desarrolla en paralelo a la vía férrea y otras carreteras perpendiculares que conectan las poblaciones ubicadas en la Cordillera Central pertenecientes al departamento de Caldas. Desde Mariquita parte la carretera que pasa por Fresno-Padua-Letras y llega hasta Manizales y desde San Felipe parte la carretera que pasa por Falan-Palocabildo-Casabianca-Padua-Herveo. Además de estas carreteras principales existen otras secundarias que articulan poblaciones menores de la zona. Completando el sistema de carreteras y red férrea, se encontraba el cable aéreo Manizales-Mariquita, el cual tenía como objetivo la comercialización del café tipo exportación producido en la zona del llamado Viejo Caldas. A pesar de su corta vida útil, el cable permitió la conexión de los territorios cafeteros de la Cordillera Central con el comercio internacional a través del Ferrocarril de La Dorada, por lo menos mientras se logró la interconexión de esta zona con el Ferrocarril del Pacífico.

La estación de pasajeros de Mariquita se encuentra ubicada a un kilómetro de la plaza fundacional en dirección suroriente. Además de la estación, el conjunto está constituido por seis volúmenes anexos, dispuestos en doble línea a lado y lado de la línea férrea. Estos volúmenes cubrian diferentes funciones como bodegas, talleres, casas de habitación en la medida en que este conjunto fue proyectado como el principal nodo del sistema. En la actualidad todo el conjunto está en desuso.

Adicionalmente, en el tramo se encuentran otras cuatro estaciones de pasajeros: estación Km 42, paradero Nuevo Méjico, San Felipe y
Guayabal. La estación Km 42 y el paradero Nuevo Méjico se encuentran ubicados en contextos rurales no urbanizados entre las estaciones de Honda y Mariquita. En cercanías al cruce de la carretera que conduce a Falan se ubica la estación San Felipe entre la carretera principal y la vía férrea; 11 kilómetros al sur se encuentra la estación Guayabal, ubicada aproximadamente a 750 metros al norte de la plaza fundacional, entre la vía principal y la línea férrea, estas conforman el borde occidental de casco urbano. Respecto al estado actual de las estaciones $\mathrm{Km}$ 42, San Felipe y Guayabal, fueron adaptadas en su interior como vivienda informal, mientras que el paradero Nuevo Méjico se encuentra en total abandono.

Con respecto al campamento Calunga, está ubicado aproximadamente a $5 \mathrm{~km}$ del casco urbano de Honda, en un sitio aislado, rural, con anexos de servicios, actualmente su uso es de vivienda colectiva.

Para sortear las fuentes hídricas de este tramo se construyeron diez puentes, de los cuales cuatro se encuentran en el tramo HondaMariquita: puente quebrada Calunga, puente

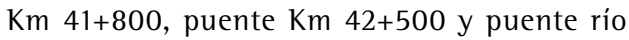
Padilla. Los otros seis se encuentran en el tramo Mariquita-Armero: puente río Lumbí 1, puente río Lumbí 2, puente quebrada el Penitente, puente río Sabandija, puente quebrada Arrayanes y puente quebrada Caimana. El puente más importante está sobre el río Sabandija consta de dos cuerpos y un total de 80 metros de longitud. Los demás puentes oscilan entre 30 metros, en quebradas y ríos, y 4 metros en el caso de las escorrentías.

El estado de conservación de las estaciones de pasajeros es en general bueno a excepción de los acabados, pintura y carpintería, aunque se debe hacer una salvedad con el paradero Nuevo Méjico, el cual se encuentra reducido a la estructura de sus muros. Los puentes que aún están en pie tienen en general un buen estado de conservación, exceptuando las traviesas y rieles; no obstante, cabe mencionar la desaparición del puente sobre el río Lumbí 1.

\section{Tramo 5: de vuelta al río, Armero a Ambalema}

El quinto y último tramo está definido desde la desaparecida población de Armero (estación 


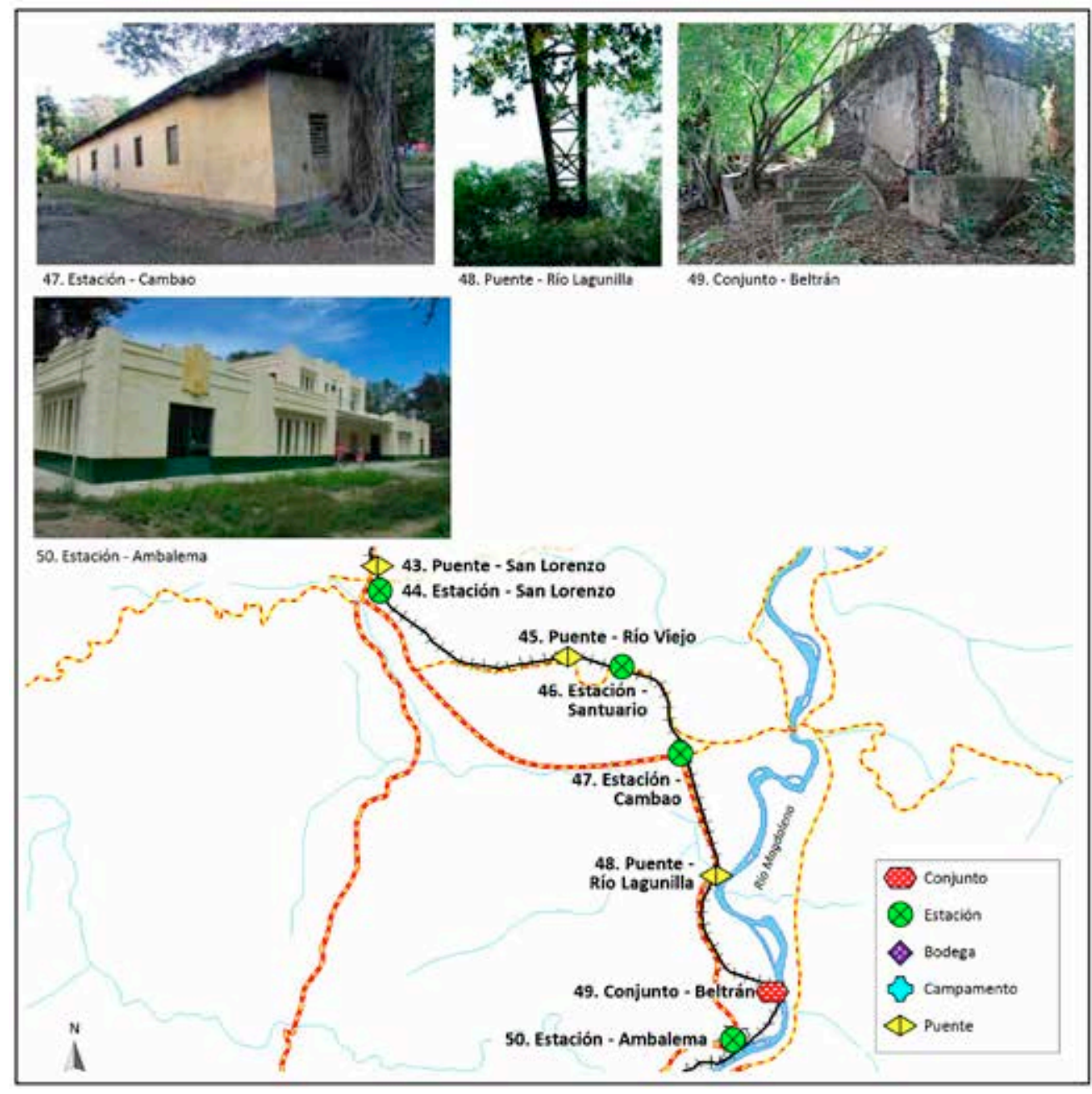

San Lorenzo) en el kilómetro 79, hasta llegar a la estación de Ambalema, final de línea, en el kilómetro 111. La línea férrea busca nuevamente el contacto directo con el río Magdalena tomando dirección suroriental por terreno plano. Aunque el paso de la línea férrea sobre el río Lagunilla se encuentra muy cercano al Magdalena, solamente a partir del antiguo puerto de Beltrancito corre por la ribera del río.

A partir de Ambalema, última estación de pasajeros de la línea del Ferrocarril de La Dorada, se inició una nueva prolongación para lograr la conexión con el Ferrocarril del Tolima (Ambalema-Buenos Aires, lbagué-Flandes) que a su vez conecta con el Ferrocarril de Girardot (Girardot-Facatativá) y este último con el Ferrocarril de la Sabana (Facatativá-Bogotá), se conforma así el sistema férreo de conexión con la capital del país. Adicionalmente, la línea se relaciona con el camino a la Sabana en el sitio de Cambao, la población de Beltrán, por ferry, en el puerto de Beltrancito, y el puerto de Gra- malotal, también vía ferry, desde la estación de Ambalema.

El trazado de la carretera principal no sigue la línea férrea pues están a una distancia aproximada de 3 kilómetros, pero articula los mismos puntos: cruce de Cambao en conexión con la carretera alterna a la Sabana y los puertos fluviales de Ambalema, Gramalotal y Beltrán. Cabe resaltar la relación que hay entre la estación de pasajeros Cambao (kilómetro 96) y el punto "cruce de la carretera hacia Cambao", puesto que en dicha población, ubicada al margen derecho del río Magdalena, confluyen varias carreteras procedentes de diferentes puntos del pais, por ejemplo, desde el occidente conecta Manizales-Líbano-Amero-Cambao, desde el norte Honda-Cambao, desde el sur GirardotCambao y desde el centro del país BogotáFacatativá-Albán-Cambao.

Beltrancito constituyó un puerto muy importante con casi $1 \mathrm{~km}$ de instalaciones portuarias a lo largo del río Magdalena, pero la
Figura 8:

Tramo 5 - Ferrocarril de La Dorada

Fuente:

Imagen y fotografías elaboradas por los autores 
prolongación del ferrocarril hasta Girardot hizo innecesaria esta parada haciéndola obsoleta, razón por la cual se levantó toda la infraestructura quedando el conjunto reducido a ruinas. En la actualidad solo se observan algunos cimientos y muros bajos en piedra ubicados sobre la margen izquierda del río, a 3 kilómetros al nororiente del casco histórico de Ambalema.

La antigua población de San Lorenzo, más conocida con el nombre de Armero, fue totalmente destruida por una avalancha de lodo por el deshielo del nevado del Ruiz que desbordó el cauce del río Lagunilla en $1985^{6}$, fue arrasada una parte importante de su casco urbano incluyendo la estación de pasajeros del ferrocarril. Esta se encontraba ubicada a dos cuadras de la plaza principal, en el borde oriental de la zona urbana, donde la línea férrea marcaba el límite del casco urbano. De esta manera, la función como nodo regional de Armero y su estación fue abruptamente rota y desplazada hacia centros vecinos.

La estación de Santuario y el puente sobre el río Viejo se ubican en un contexto rural no urbanizado, a medio camino entre San Lorenzo y Cambao. De la esta estación y del puente no se obtuvieron registros por las dificultades de acceso a la zona. Cabe resaltar que se encontraron registros aerofotográficos de 1941 que permiten afirmar que la estación Santuario servía a un complejo industrial que desapareció totalmente del paisaje. Esto constituye una de las transformaciones más drásticas de los elementos anexos al ferrocarril.

La estación de Cambao está ubicada en un

El 13 de noviembre de 1985, a las 11:30 de la noche, una avalancha del río Lagunilla, ocasionada por la erupción del volcán Arenas del nevado del Ruiz, borró del mapa a Armero, la más importante ciudad de norte del Tolima, que dejó un saldo de 26.000 muertos (el $65 \%$ de las muertes ocasionadas ese año por desastres de la naturaleza), 20.611 damnificados y heridos, muchos de ellos mutilados y gravemente afectados, por no decir que derrumbados psicológicamente, e incalculables pérdidas económicas (4.400 viviendas, 19 puentes, $\$ 1.400$ millones del comercio). Era la tragedia de mayor magnitud en la historia de Colombia (Rueda, 1999). contró entre las ruinas de la antigua población, con 7 metros de luz, la estructura perdura hasta hoy a pesar de la avalancha. Al puente sobre el río Viejo, ubicado en el sector de Santuario, no se tuvo acceso por problemas de accesibilidad a la zona. Y finalmente, el puente sobre el río Lagunilla, ubicado entre las estaciones de San Lorenzo y Beltrán, con 15 metros de luz, se encuentra en regular estado de conservación, pero mantiene en pie sus bases y estructura.

En este tramo, de Armero a Ambalema, el territorio ha tenido la transformación más drástica. La desaparición de la población de Armero marca esta dinámica, así como la desaparición de la actividad industrial alrededor de la estación Santuario y la del puerto de Beltrancito. Esto implica el cierre de ciclos de productividad relacionados directamente con el ferrocarril y la transformación de las dinámicas territoriales de toda la zona.

\section{Conclusiones}

Por la inactividad de la línea del Ferrocarril de La Dorada, que ya supera los 35 años, sus diferentes elementos constitutivos, todos con funciones especializadas, se han desarticulado, por lo cual, se ha perdido la noción de sistema. Esta situación coyuntural ha estimulado la pérdida de elementos vitales como: puentes (puente quebrada Humareda y el puente Pearson, entre otros), tramos de rieles levantados y estaciones destruidas (Santuario o Nuevo México). A pesar de las condiciones señaladas, la línea guarda un alto nivel de integridad si se toman consideraciones como la integración al paisaje, la conservación de los terraplenes y, como condición vital para la viabilidad de proyectos de reestructuración del Ferrocarril, el respeto y bajo nivel de invasión del corredor férreo. En general, la franja de terreno reservada al ferrocarril está despejada, accesible y sin problemas visibles de ocupación o invasión de terrenos, excepto en el tramo ubicado en las inmediaciones de la hacienda Santuario, de aproximadamente 7 $\mathrm{km}$, y el tramo que atravesaba el antiguo casco urbano de Armero.

El estado de conservación de los diferentes elementos constitutivos del ferrocarril es variado y relativo a consideraciones y circunstancias puntuales de cada uno. Es evidente que la falta de mantenimiento y el desuso atacan por igual a todos los elementos de la línea: en puentes y 
tendido de rieles es claro el deterioro por fatiga de materiales, desaparición de traviesas de madera, levantamiento de rieles y la pérdida total y/o el colapso de estructuras como la ya mencionada del puente Pearson. Las estaciones y bodegas aisladas, ubicadas generalmente en zonas rurales, tienen dificultades para insertarse dentro de la lógica territorial de su contexto. Con respecto a los nodos principales o conjuntos, su situación es precaria, pues se debaten entre la desaparición por obsolescencia como el caso de Beltrancito, el uso parcial (La Dorada), la ocupación de áreas (Honda) o el cierre total con vigilancia (Mariquita). Cualquiera de estas opciones sigue generando deterioro integral para las áreas del ferrocarril y su entorno urbano.

Del total de 50 elementos registrados, el 58\% corresponde a elementos de circulación, $38 \%$ a transferencia y apenas un $4 \%$ a las funciones complementarias. De estos 50 elementos, 19 están en excelente o buen estado de conservación, repartidos equitativamente entre las funciones de circulación y transferencia. De estos 19, un total de 12 elementos están concentrados en los tramos 3 y 4 , que corresponden al área urbana de Honda y se extiende hasta la desaparecida ciudad de Armero. En la medida que en estos tramos la carretera corre en paralelo y muy cerca de la línea férrea, se considera que la vitalidad aportada por el corredor ha tenido un efecto positivo sobre el estado de conservación de dichos elementos.

En regular estado de conservación se encontraron 17 elementos, repartidos entre las funciones de circulación (10), de transferencia (5) y complementarios (2). De estos, 12 están concentrados en los tramos 1 y 4 . Esta concentración se debe a las condiciones hidrográficas de los tramos que exigieron la construcción de puentes a los cuales no se les ha realizado ningún mantenimiento en los últimos 35 años.

De otra parte, de los 50 elementos, 12 están catalogados como desaparecidos o en mal estado de conservación: 8 elementos corresponden a la función de circulación, y los 4 restantes, a la función de transferencia, puntualmente son: el paradero de Nuevo México y el conjunto de Beltrán, ambos en mal estado de conservación, y las estaciones de San Lorenzo y Santuario, desaparecidas. Adicionalmente, de los 8 elementos de circulación, 4 están concentrados en el tramo 3, específicamente al casco urbano de Honda (en una longitud de apenas $4 \mathrm{~km}$ aproximadamente). Este precario estado de conservación está relacionado con el crecimiento urbano de la ciudad, con la adaptación al tráfico vehicular y a razones históricas de desaparición de la línea original.

Por su función primordial, el estado de conservación de los elementos de circulación es el primer factor responsable de la discontinuidad total de la línea del Ferrocarril de La Dorada. De los 21 elementos registrados en esta función solo 9, el 31\%, se encuentran en un buen estado de conservación. De acuerdo con lo anotado en el inicio del registro de los tramos, esta calificación de "buen estado" no se refiere a su resistencia y desempeño estructural, sino a la integridad de sus elementos constitutivos. Esto quiere decir que el 69\% de los elementos presentan regular o mal estado de conservación, o definitivamente están desaparecidos. El balance para el total de la línea da cuenta del abandono generalizado, factor que obstaculiza cualquier proyecto de revitalización y reapertura de la línea férrea.

El balance respecto al estado del conjunto de elementos de la función de transferencia y complementarios es un poco más alentador. De los 21 elementos registrados, el 49\%, o sea 10, tienen un excelente o buen estado de conservación. En este grupo se encuentran las estaciones de inicio y final del tramo, La Dorada y Ambalema, que están a cargo de las administraciones locales y han sido intervenidas para integrarlas a funciones colectivas. Un total de 10 estaciones de pasajeros han sido ocupadas como viviendas informales, lo que genera mínimos cuidados que se ven reflejados en mejor estado de conservación en tanto los ocupantes cuidan las condiciones básicas de habitabilidad (techo sin goteras, ventanas, etc.).

Respecto a las dos estaciones desaparecidas, la de San Lorenzo lo fue por desastre natural, sin vestigios o huellas en el sitio, y la de Santuario, de manera intencional por cambios de uso del suelo dentro de la hacienda Santuario. Las estaciones de Nuevo México y Beltrán presentan un mal estado de conservación por pérdida de su función en el territorio. Cambios en la vocación del territorio y la circunstancia de no estar vinculadas a un casco urbano dejan a estas estaciones en una posición muy vulnerable, con pocas opciones de encontrar otra función. Los campamentos registrados (Calunga y Caporal) 
por su estructura arquitectónica tienden a ser usados para vivienda, pero en la modalidad de inquilinato, lo que pone en duda sus opciones de conservación a futuro.

El caso de la estación y conjunto de Mariquita genera un interés particular pues está en total desuso, cerrada, "cuidada" contra posibles invasiones o apropiaciones ilegales, pero sin ningún servicio a la comunidad. Este nodo que fue pensado como el punto central de toda la línea, con interconexiones multimodales y gran impacto regional, representa en la actualidad un vacío urbano de difícil tratamiento.

El Ferrocarril de La Dorada ocupa un lugar poco relevante en la memoria colectiva de las poblaciones asociadas. Para la población joven representa una infraestructura inservible, sin relación con una experiencia directa, mientras los mayores, quienes lo vivieron, ven al ferrocarril revestido de la nostalgia que evoca una época de prosperidad y movimiento, relacionado con un mejor vivir y con mayores oportunidades para todos.

En cuanto al valor patrimonial de la línea, el estudio permitió definir las siguientes consideraciones:

1. La relevancia del Ferrocarril de La Dorada para la construcción de territorio, de manera multiescalar, lo que permitió el desarrollo de zonas rurales y urbanas a partir de su relación con vecinos cercanos y lejanos y el intercambio constante de mercancías y pasajeros.

2. Valor en tanto huella de la llegada de la modernidad y la industrialización a una zona del país con una gran importancia, pero también con un gran retraso en términos tecnológicos.

3. El desarrollo de formas multimodales de transporte que el sistema propició, que fue la característica principal del Ferrocarril de La Dorada y de los ferrocarriles en Colombia en las primeras etapas de su conformación y desarrollo.
4. Sistema privilegiado para contar la historia de la conformación y modernización del país en todas sus etapas: los esfuerzos para la conformación, los errores en los modelos de gestión y contratación utilizados, los retos técnicos para lograr avances significativos, el desplazamiento por otras formas de transporte, el angustioso ocaso y finalmente la inactividad total y el desmantelamiento del sistema.

5. Valor común a otros sistemas de transporte, referido a su capacidad de construcción y particularización de paisaje a partir de la inserción de elementos variados como los terraplenes, puentes, viaductos y demás elementos, que combinan las condiciones naturales del espacio geográfico con el trabajo humano, la cultura, la velocidad y la huella cultural.

\section{Referencias}

Archivo General de la Nación (1907). Sección República. Archivo Histórico de Ferrocarriles Nacionales de Colombia.

Bateman, A. (2005). Historia de los ferrocarriles de Colombia. Bogota: Sociedad Colombiana de Ingenieros.

Duque, G. (2013). Universidad Nacional de Colombia. Repositorio UN. Obtenido de La navegación del Magdalena y la conurbación Honda - La Dorada: http://www.bdigital. unal.edu.co/9422/

Ortega, A. (1911). Informe al Ministerio de Obras Públicas. Bogotá: Archivo General de la Nación.

Poveda, G. (2010). Carrileras y locomotoras Historia de los ferrocarriles en Colombia. Medellín: Fondo Editorial Universidad EAFIT.

Rueda, J. E. (1999). La avalancha de Armero: noviembre 13 de 1985. Revista Credencial Historia, 117.

Sánchez, E. (2014). Codazzi y el salto de Honda. Revista Credencial Historia, 289. 
